\title{
The Role of Previous Therapies and Sites of Metastasis as Influencing Factors on Discordance of ER, PR and HER2 Status Between Primary and Metastasized Breast Cancer
}

\author{
CONSTANZE VOGEL ${ }^{1}$, WOLFRAM MALTER ${ }^{1}$, BERND MORGENSTERN ${ }^{1}$, \\ SEBASTIAN LUDWIG ${ }^{1}$, JÖRG JANNE VEHRESCHILD ${ }^{2,3}$, STEFANIE HAMACHER ${ }^{4}$, \\ PETER MALLMANN $^{1}$, VERENA KIRN $^{5 *}$ and FABINSHY THANGARAJAH ${ }^{1 *}$ \\ ${ }^{1}$ Department of Obstetrics and Gynecology, Medical Faculty, University Hospital of Cologne, Cologne, Germany; \\ ${ }^{2}$ Department I for Internal Medicine, University Hospital of Cologne, Cologne, Germany; \\ ${ }^{3}$ German Centre for Infection Research, partner-site Bonn-Cologne, Cologne, Germany; \\ ${ }^{4}$ Department of Medical Statistics, Informatics and Epidemiology, University of Cologne, Cologne, Germany; \\ ${ }^{5}$ Department of Senology, Heilig Geist Krankenhaus, Cologne, Germany
}

\begin{abstract}
Background/Aim: The aim of the present study was to analyze metastasized breast cancer (BC) patients with regard to the discordance of estrogen receptor $(E R)$, progesterone receptor $(P R)$ and human epidermal growth factor receptor 2 (HER2). We especially aimed to analyze the association between the change of tumor biology and previous treatment or metastatic sites. Patients and Methods: Patients with metastasized $B C$ who were treated at the Department of Gynecology/Breast Center of the University Hospital of Cologne were analyzed. Results: Loss of HER2 occurred more frequently in lymph node metastases that were not in the axillary region $(p=0.026)$. Letrozole showed a significant correlation with loss of ER and/or PR $(p=0.041)$. Improved overall survival and post-metastasis survival were noticed with a gain of HER2 ( $p=0.044$ and $p=0.009$, respectively) and concordant positive $E R$ and $P R$ status ( $p=0.002$ and $p=0.001$, respectively). Conclusion: The discordance of receptors and the dependence of $B C$ on therapies as well as metastatic sites stresses the necessity of early sample taking to offer patients suitable therapy options.
\end{abstract}

\footnotetext{
*These Authors contributed equally to this study.

Correspondence to: Fabinshy Thangarajah, MD, University Hospital of Cologne, Department of Obstetrics and Gynecology, and Breast Center, Medical Faculty, Kerpener Strasse 34, 50931 Cologne, Germany. Tel: +49 22147886545, e-mail: Fabinshy.Thangarajah@ukkoeln.de
}

Key Words: Metastasized breast cancer, discordance of receptors, overall survival.
Breast cancer (BC) mortality rates have been declining since the 1990s. Nevertheless, it is still the second leading cause of cancer-related deaths in women in high developed and the first cause in low developed countries. In 2015, 523,000 women and 10,000 men died because of breast cancer (1-3).

In total, $20-30 \%$ of women with early $\mathrm{BC}$ show metastases (2). At the time of diagnosis of primary as well as metastasized BC, histological analyses of the specimens are important for choosing treatment, since the possibility of individual therapy is dependent on the hormone receptors (HR) [estrogen receptor (ER), progesterone receptor (PR)] and the human epidermal growth factor receptor 2 (HER2) status (4). Discordance of tumor biology (ER, PR, HER2) has been discussed in numerous studies since the late 70s (57). A meta-analysis of 48 studies (years 1983-2012) showed discordances for ER, PR and HER2 of $20 \%, 33 \%$ and $8 \%$, respectively (5). The importance to retake biopsies for histological analysis at the time of diagnosis of metastases was a point of interest in previous studies (8-10). Some studies show improved survival after taking a biopsy. One reason for the association between carrying out a biopsy and improved survival is the finding of false-tested primary tumor's biology, which could support the decision to change and improve treatment (8). Sachar et al. have illustrated the association between improved survival and a biopsy which either confirmed prior ER, PR and HER2 status or completed missing data of initial diagnosis (9). Tobin et al. have reported the significant influence of molecular subtype and tumor characteristics assessed by gene expression on postrelapse survival (10). Various other authors have especially illustrated the association of tumor biology's discordance and worse overall survival (OS) $(5,7,11)$. Although there are different theories and studies, the exact reason for a change 
Table I. Characteristics of primary BC.

\begin{tabular}{|c|c|c|c|c|c|c|c|}
\hline \multirow{2}{*}{$\begin{array}{l}\text { Characteristics } \\
\text { Age (years) }\end{array}$} & & \multicolumn{2}{|c|}{ Total $(n=222)$} & \multicolumn{2}{|c|}{ Discordance $(\mathrm{n}=115)$} & \multicolumn{2}{|c|}{ Concordance $(n=107)$} \\
\hline & & 52.4 & \pm 11.8 & 52.2 & \pm 11.5 & 52.6 & \pm 12.1 \\
\hline & Neoadjuvant & 56 & 25.6 & 35 & 30.4 & 21 & 20.2 \\
\hline & Adjuvant & 114 & 52.1 & 56 & 48.7 & 58 & 55.8 \\
\hline & Primary metastasized & 49 & 22.4 & 24 & 20.9 & 25 & 24.0 \\
\hline \multirow[t]{3}{*}{ Receptors } & ER positive & 179 & 80.6 & 94 & 81.7 & 85 & 79.4 \\
\hline & PR positive & 157 & 70.7 & 82 & 71.3 & 75 & 70.1 \\
\hline & HER2 positive & 43 & 19.4 & 27 & 23.5 & 16 & 15.0 \\
\hline \multirow[t]{4}{*}{ Subgroups } & 1: ER/PR+, Her2- & 148 & 66.7 & 73 & 63.5 & 75 & 70.1 \\
\hline & 2: ER/PR+, Her2+ & 36 & 16.2 & 26 & 22.6 & 10 & 9.3 \\
\hline & 3: ER/PR-, Her2+ & 7 & 3.2 & 1 & 0.9 & 6 & 5.6 \\
\hline & 4: ER/PR-, Her2- & 31 & 14.0 & 15 & 13.0 & 16 & 15.0 \\
\hline \multirow[t]{7}{*}{ pT } & Tis & 38 & 17.1 & 15 & 14.0 & 23 & 20.0 \\
\hline & $0 / \mathrm{mic}$ & 8 & 3.8 & 4 & 3.5 & 4 & 4.0 \\
\hline & 1 & 75 & 35.6 & 34 & 29.6 & 41 & 41.0 \\
\hline & 2 & 88 & 41.7 & 55 & 49.5 & 33 & 33.0 \\
\hline & 3 & 23 & 10.9 & 13 & 11.7 & 10 & 10.0 \\
\hline & 4 & 17 & 8.16 & 5 & 4.35 & 12 & 12.0 \\
\hline & Unknown & 11 & & 4 & & 7 & \\
\hline \multirow[t]{5}{*}{$\mathrm{pN}$} & 0 & 71 & 35.3 & 34 & 32.7 & 37 & 3.1 \\
\hline & 1 & 77 & 38.3 & 38 & 36.5 & 39 & 40.2 \\
\hline & 2 & 32 & 15.9 & 19 & 54.8 & 13 & 13.4 \\
\hline & 3 & 21 & 10.4 & 13 & 12.5 & 8 & 8.2 \\
\hline & Unknown & 21 & & 11 & & 10 & \\
\hline \multirow[t]{4}{*}{ G } & 1 & 5 & 2.8 & 4 & 4.3 & 1 & 1.1 \\
\hline & 2 & 116 & 64.4 & 58 & 63 & 58 & 65.9 \\
\hline & 3 & 59 & 32.8 & 30 & 32.6 & 29 & 33.0 \\
\hline & Unknown & 42 & & 20 & & 19 & \\
\hline \multirow[t]{4}{*}{ Primary operation } & Breast conserving therapy & 116 & 54.0 & 63 & 57.3 & 53 & 50.5 \\
\hline & Mastectomy & 81 & 37.7 & 42 & 38.2 & 39 & 87.6 \\
\hline & None & 18 & 8.4 & 5 & 4.5 & 13 & 12.4 \\
\hline & Unknown & 7 & & 5 & & 2 & \\
\hline \multirow[t]{4}{*}{$\mathrm{LN}$ treatment } & SNB & 37 & 17.5 & 17 & 15.3 & 20 & 20.0 \\
\hline & $\mathrm{AD}$ & 152 & 72.0 & 86 & 77.5 & 66 & 66.0 \\
\hline & None & 22 & 10.4 & 8 & 7.2 & 14 & 14.0 \\
\hline & Unknown & 11 & & 4 & & 7 & \\
\hline
\end{tabular}

LN: Lymph node; SNB: sentinel node biopsy; AD: axillary dissection.

of tumor biology is still not clear. On the one hand the less than perfect reproducibility is caused by preanalytic and analytic variables of receptor determination. On the other hand, further possibilities mentioned are heterogeneity of primary $\mathrm{BC}$, therapy induced clonal selection or a change of the tumors' geno- or phenotype $(7,12,13)$.

After discovering any discordance, patient's treatment can be changed. A prospective study observed a change of patients' treatment in $14 \%$ of cases, whereas no difference of survival was seen between the group with concordant and the group with discordant ER, PR or HER2 (14).

However, there are still no standardized clinical guidelines as to whether or not to alter treatment after the diagnosis of a change in tumor biology (4). Nevertheless, it was decided to recommend the reassessment of HR and HER2 status by taking a biopsy of newly-diagnosed metastases (4).
The aim of the present study was to analyze patients with metastasized BC concerning the discordance of ER, PR and HER2. Especially the association between the change of tumor biology and either previous treatment (chemotherapy, endocrine therapy and antibody therapy with trastuzumab/ pertuzumab) or metastasis sites was questioned. Furthermore, it was examined whether or not overall survival (OS) is influenced by the discordance of ER, PR and HER2.

\section{Materials and Methods}

Patient characteristics. A total of 460 patients with metastasized BC that were treated at the Department of Gynecology/Breast Center of the University Hospital of Cologne between 2013 and 2016 were identified. Data were collected using the ClinicalSurveys.net documentation platform of the University Hospital of Cologne in cooperation with QuestBack GmbH, Cologne, Germany. 
Table II. Metastasis sites and treatment until second biopsy.

\begin{tabular}{lrrrrrr}
\hline Metastasis & & & & & & \\
\hline Metastasis sites & 15 & 6.8 & 8 & 7.0 & 7 & 6.5 \\
Lung & 53 & 23.9 & 27 & 23.5 & 26 & 24.3 \\
Liver & 9 & 4.1 & 6 & 5.2 & 3 & 2.8 \\
Brain & 54 & 24.3 & 28 & 24.3 & 26 & 24.3 \\
Bone & 9 & 4.1 & 3 & 2.6 & 6 & 5.6 \\
Pleura aspirate & 8 & 3.6 & 4 & 3.5 & 4 & 3.7 \\
Pleura biopsy & 5 & 2.3 & 3 & 2.6 & 2 & 1.9 \\
LN ax & 19 & 8.6 & 13 & 11.3 & 6 & 5.6 \\
LN other & 2 & 0.9 & 2 & 1.7 & 0 & 0.0 \\
Ascites & 2 & 0.9 & 1 & 0.9 & 1 & 0.9 \\
Peritoneum & 23 & 10.4 & 8 & 7.0 & 15 & 14.0 \\
Thoracic wall & 2 & 0.9 & 1 & 0.9 & 1 & 0.9 \\
Breast & 2 & 0.9 & 1 & 0.9 & 1 & 0.9 \\
Intestinum & 2 & 8.6 & 10 & 8.7 & 9 & 8.4 \\
Other & & & & & & \\
Treatment & 153 & 68.9 & 87 & 75.7 & 66 & 61.7 \\
Chemotherapy & 153 & 68.9 & 86 & 74.8 & 67 & 62.6 \\
Endocrine therapy & 57 & 25.7 & 36 & 31.3 & 21 & 19.6 \\
Antibodies & 31 & 14.0 & 22 & 19.1 & 9 & 8.4 \\
Trastuzumab/Pertuzumab & 28 & 12.6 & 71 & 61.7 & 62 & 58.5 \\
Radiation therapy & & & & & & \\
\hline
\end{tabular}

ax: Axillary.

The following patients' data were abstracted from electronic and non-electronic records:

A) primary BC: sex, dates of birth and of diagnosis; histological grade (G1-3). B) pTNM classification; tumor biology (ER, PR, HER2); metastasized BC: date of biopsy and site of metastasis; received treatment; tumor biology. C) date of death or last contact.

Ethical statement. This was a secondary use real-life data collection of retrospective data. All study investigators are directly involved in patient care at the University Hospital of Cologne. No interventions were performed as part of this epidemiological cohort study. Data collection and storage were performed on site by site personnel using current techniques of privacy assurance. In this situation, according to $\$ 15$ subparagraph 1 "Berufsordnung für die nordrheinischen Ärztinnen und Ärzte" (code of medical ethics for physicians in the state of Northrhine-Westfalia) and §6 subparagraph 2 "Gesundheitsdatenschutzgesetz - GDSG NRW" (health privacy law for the state of Northrhine-Westfalia) no formal vote by an Ethics Committee or informed consent was necessary for this study.

Ethical approval. All procedures performed in studies involving human participants were in accordance with the ethical standards of the institutional and/or national research committee and with the 1964 Helsinki declaration and its later amendments or comparable ethical standards.

Statistical analysis. First, a descriptive analysis of patients' characteristics was performed. Data are presented as mean \pm standard deviation (SD), mean (interquartile range) or count (percentage), respectively. Mc Nemar's test was calculated to analyze variations of the immunophenotype in primary and metastasized situations. Second, univariate logistic regression was performed to analyze possible association of various factors including age, interval from primary to metastasized cancer, treatment and metastasis sites on discordant tumor biology. Every factor, which reached a $p$-value $<0.1$ was entered in a multivariate logistic regression model, where $p$-values $<0.05$ were defined as statistically significant. Results are presented as odds ratio (OR), corresponding 95\% confidence interval $(\mathrm{CI})$ and $p$-value.

Furthermore, prognosis of different patients' subgroups was investigated. OS was defined as time from primary diagnosis to death, PMS (post-metastasis-survival) as time from diagnosis of metastasis to death. A Cox regression was performed and results are given as hazard ratio (HR), corresponding 95\% CI and $p$-value. Kaplan-Meier plots are used for visualization of group differences.

Statistical analysis was done using SPSS Statistics for Windows, version 25 (IBM Corp., Armonk, NY, USA).

\section{Results}

Of the 460 identified patients, 222 patients were included. Four patients were excluded because of male gender, another four due to their diagnosis of a second malignancy and 232 patients because of incomplete data.

Within the 222 included patients, median age at primary diagnosis was 51 years, ranging from 22 to 84 years. Median survival was 6 years (77 months), whereas median duration of the interval between primary and secondary biopsy was 52 months. After the diagnosis of metastasized breast cancer, median survival was 18 months. Patient's and tumor characteristics are illustrated in Tables I and II.

Total discordance of ER, PR and HER2 was 18\%, 34\% and $14 \%$ (Table III). ER loss (25 patients) was observed more frequently than ER gain (16 patients), same situation was observed for PR (79\% positive to negative, $21 \%$ negative to positive). Furthermore, 30 patients (14\%) changed their HER2 statuses until they reached a metastasized situation (primary diagnosis 19\% HER2 positive; change in 14 patients from positive to negative, and 16 from negative to positive).

The McNemar's test only showed significant differences between loss and gain for PR $(p<0.001)$.

According to the expression of ER, PR and HER2, patients were divided into four subgroups (definitions of subgroups are illustrated in Table IV). A change of tumor phenotype was observed in 29\% (63 patients), while a loss of HR (change of group $1 / 2$ to group $3 / 4$ ) was noticed in $12.5 \%$ (Table IV).

Discordance of tumor phenotype. First, the association between different subgroup concordance/discordance combinations and treatment, age of diagnosis, interval of primary to secondary biopsy, as well as metastasis sites were tested in a univariate regression analysis. A $p$-value $<0.1$ was calculated for many factors. Therefore, detailed results of univariate analyses are illustrated in Table V.

After entering those factors in multivariate analysis, $p$-values $<0.05$ were considered statistically significant 
Table III. Tumor biology of primary and metastasized BC.

\begin{tabular}{|c|c|c|c|c|c|c|}
\hline \multirow[b]{2}{*}{ Primary } & \multicolumn{2}{|c|}{$\begin{array}{l}\text { ER discordance } \\
\text { (222 Patients) }\end{array}$} & \multicolumn{2}{|c|}{$\begin{array}{l}\text { PR discordance } \\
\text { (221 Patients) }\end{array}$} & \multicolumn{2}{|c|}{$\begin{array}{l}\text { HER2 discordance } \\
\text { (217 Patients) }\end{array}$} \\
\hline & \multicolumn{2}{|c|}{ Metastasis } & \multicolumn{2}{|c|}{ Metastasis } & \multicolumn{2}{|c|}{ Metastasis } \\
\hline & + & - & + & - & + & - \\
\hline+ & 154 & 25 & 97 & 60 & 28 & 14 \\
\hline- & 16 & 27 & 16 & 48 & 16 & 159 \\
\hline Total discordance & \multicolumn{2}{|c|}{$41(18.5 \%)$} & \multicolumn{2}{|c|}{$76(34.4 \%)$} & \multicolumn{2}{|c|}{$30(13.8 \%)$} \\
\hline$p$-Value & \multicolumn{2}{|c|}{0.221} & \multicolumn{2}{|c|}{$<0.001$} & \multicolumn{2}{|c|}{0.856} \\
\hline
\end{tabular}

Table IV. Tumor phenotype (primary and metastasized BC).

\begin{tabular}{|c|c|c|c|c|c|c|}
\hline \multirow[t]{2}{*}{ Subtype/Primary diagnosis } & \multirow[t]{2}{*}{ No. of cases } & \multicolumn{4}{|c|}{ Metastasis } & \multirow[t]{2}{*}{ Discordance $\%$} \\
\hline & & Group 1 & Group 2 & Group 3 & Group 4 & \\
\hline Group 1: ER/PR+, Her2- & 144 & $115(77.7 \%)$ & $13(8.7 \%)$ & 0 & $16(10.8 \%)$ & $29(20.1 \%)$ \\
\hline Group 2: ER/PR+, Her2+ & 35 & $11(30.6 \%)$ & $17(47.2 \%)$ & $5(13.9 \%)$ & $2(5.6 \%)$ & $18(51.4 \%)$ \\
\hline Group 3: ER/PR-, Her2+ & 7 & $1(14.3 \%)$ & 0 & $6(85.7 \%)$ & 0 & $1(14.3 \%)$ \\
\hline Group 4: ER/PR-, Her2- & 31 & $12(38.7 \%)$ & $2(6.5 \%)$ & $1(3.2 \%)$ & $16(51.6 \%)$ & $15(48.4 \%)$ \\
\hline Total & 217 & 139 & 32 & 12 & 34 & $29.0 \%$ \\
\hline
\end{tabular}

(Table VI). Less concordance of positive HR (group 1 and $2)$ seems to be associated with any chemotherapy $(p<0.001)$, whereas endocrine therapy (ET) affects HR concordance positively $(p<0.001)$.

The possibility of first any group discordance $(p=0.002)$, second the loss of HR (group $1 / 2$ to $3 / 4 ; p=0.038$ ) as well as third the loss of HER2 (group $2 / 3$ to $1 / 4 ; p<0.001$ ) were calculated for trastuzumab/pertuzumab (T/P).

Furthermore, loss of HER2 seems to occur more frequently in lymph node metastases that are not in the axillary region $(p=0.026)$. None of the factors showed a significant correlation with the gain of HR or HER2.

Discordance of ER and PR. Additionally, ER and PR discordance were analyzed in detail. ER as well as PR concordance were observed in 51\% (113 patients). Loss of PR was, as mentioned above, the most frequent change (27\%; 60 patients). A total of 43 patients $(19 \%)$ showed a PR loss as well as ER concordance.

Results of univariate analyses showing $p$-values $<0.1$ are illustrated in the Table VII. $p$-Values $<0.05$ were regarded as statistically significant after entering those factors into multivariate analysis (Table VIII).

In multivariate analyses, anthracyclines seemed to be associated with less concordance of ER and/or PR $(p=0.002)$. PR loss, while ER remains positive, as well as
ER and/or PR loss, presented both a significant association with ET (1: $p=0.001 \mid 2: p=0.002)$.

Especially letrozole showed a significant correlation with loss of ER and/or PR ( $p=0.041)$. Interestingly, ER concordance was observed more frequently during treatment under tamoxifen ( $p=0.041)$. Fulvestrant was associated with less ER and/or PR concordance $(p=0.015)$. Furthermore, treatment under T/P correlated with a higher probability of ER loss $(p=0.038)$. Thoracic wall metastases were found to retain ER and PR status from primary carcinoma $(p=0.033)$.

Survival. Finally, the present study showed worse OS for subgroup $4(p=0.017)$ and patients with positive PR status at primary BC $(p=0.004)$. Worse PMS was also observed for subgroup 4 ( $p=0.037)$, patients with ER and/or PR loss, as well as patients with ER concordance with PR loss ( $p=0.044$ and 2: $p=0.013$, respectively). PR status at primary $\mathrm{BC}$ was another negative prognostic factor for PMS $(p=0.044)$.

In contrast, improved OS and PMS were noticed for a gain of HER2 ( $p=0.044$ and $p=0.009$, respectively) as well as concordant positive ER and PR status $(p=0.002$ and $p=0.001$, respectively). HER2 discordance only showed significantly improved PMS $(p=0.005)$.

In addition, multivariate analysis of cox regression presented worse PMS for the group of ER concordance and 


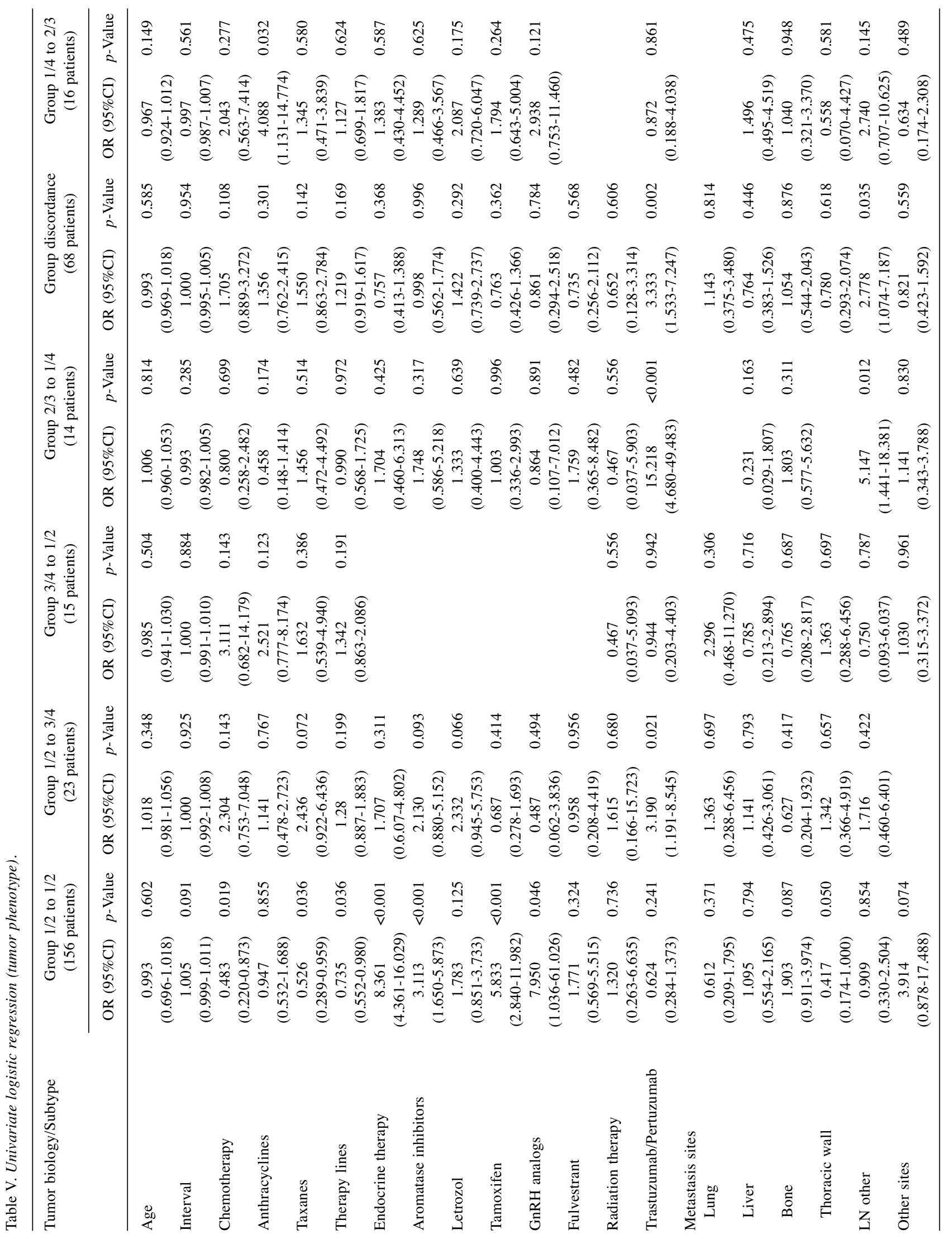


Table VI. Results of multivariate logistic regression (tumor phenotype change).

\begin{tabular}{|c|c|c|c|c|c|c|c|c|c|c|c|c|}
\hline \multirow[t]{2}{*}{$\begin{array}{l}\text { Tumor biology/ } \\
\text { Subtype }\end{array}$} & \multicolumn{2}{|c|}{$\begin{array}{l}\text { Group } 1 / 2 \text { to } 1 / 2 \\
\text { (156 patients) }\end{array}$} & \multicolumn{2}{|c|}{$\begin{array}{l}\text { Group } 1 / 2 \text { to } 3 / 4 \\
\text { (23 patients) }\end{array}$} & \multicolumn{2}{|c|}{$\begin{array}{l}\text { Group } 3 / 4 \text { to } 1 / 2 \\
\text { (15 patients) }\end{array}$} & \multicolumn{2}{|c|}{$\begin{array}{l}\text { Group } 2 / 3 \text { to } 1 / 4 \\
\text { (14 patients) }\end{array}$} & \multicolumn{4}{|c|}{$\begin{array}{l}\text { Group discordance Group } 1 / 4 \text { to } 2 / 3 \\
\text { (68 patients) }\end{array}$} \\
\hline & $\begin{array}{c}\mathrm{OR} \\
(95 \% \mathrm{CI})\end{array}$ & $p$-Value & $\begin{array}{c}\mathrm{OR} \\
(95 \% \mathrm{CI})\end{array}$ & $p$-Value & $\begin{array}{c}\mathrm{OR} \\
(95 \% \mathrm{CI})\end{array}$ & $p$-Value & $\begin{array}{c}\mathrm{OR} \\
(95 \% \mathrm{CI})\end{array}$ & $p$-Value & $\underset{(95 \% \mathrm{CI})}{\mathrm{OR}}$ & $p$-Value & $\begin{array}{c}\mathrm{OR} \\
(95 \% \mathrm{CI})\end{array}$ & $p$-Value \\
\hline Chemotherapy & $\begin{array}{c}0.189 \\
(0.079-0.453)\end{array}$ & $<0.001$ & & & & & & & & & & \\
\hline Endocrine therapy & $\begin{array}{c}13.620 \\
(6.291-29.487)\end{array}$ & $<0.001$ & & & & & & & & & & \\
\hline $\begin{array}{l}\text { Trastuzumab/ } \\
\text { Pertuzumab }\end{array}$ & & & $\begin{array}{c}3.190 \\
(1.191-8.545)\end{array}$ & 0.021 & & & $\begin{array}{c}15.491 \\
(4.570-52.514)\end{array}$ & $<0.001$ & $\begin{array}{c}3.333 \\
(1.533-7.247)\end{array}$ & 0.002 & & \\
\hline $\begin{array}{l}\text { Metastasis site; } \\
\text { LN (other than } \\
\text { axillary) }\end{array}$ & & & & & & & $\begin{array}{c}5.376 \\
(1.220-23.685)\end{array}$ & 0.026 & & & & \\
\hline
\end{tabular}

PR loss as well as HER2 at primary BC $(p=0.008$ and $p=0.010$, respectively). $\mathrm{PR}$ status at primary $\mathrm{BC}$ was a negative prognostic factor for OS $(p=0.038)$ (Tables IX-XII and Figures 1-3).

\section{Discussion}

In this retrospective study, $\mathrm{BC}$ patients were analyzed with attention to the influence of several factors on the discordance of the tumor's phenotype from the primary to the metastasized breast cancer disease. Of special interest was the association between previous treatment, metastatic sites as well as OS/PMS and the change of tumor biology.

Regarding the percentage of patients that experienced a change of receptors from primary breast cancer to metastasized breast cancer disease, previous publications have showed corresponding results to our study, which were $18 \%, 34 \%$ and $14 \%$ for ER, PR and HER2, respectively. A meta-analysis of 48 studies (years 1983-2012) showed discordances for ER, PR and HER2 in 20\%, 33\% and 8\%, respectively, whereas cases with a change of HER2 status were reported up to $24 \%$ (5). As consistent with other studies, changes of positive to negative HR (35\%) versus negative to positive $(19 \%)$ were perceived more frequently in our study $(5,7,15)$, as well as a change of PR was observed more often than an ER change $(16,13,17,11)$. Results of the subgroup analysis (group discordance in 29\%) were similar with those shown by previous studies, for example a recent study of Shiino et al. (21\% group discordance, $14 \%$ HR loss) or Nishimura et al. (25\% group discordance) $(15,18)$.

Interestingly, at primary diagnosis 28 cases were tested HR negative and 16 HER2 negative, although the corresponding relapse was tested positive. Gain of HR or HER2 has also been mentioned by other authors $(16,17,19)$. Various explanations for a discordant receptor status include either technical or genetic reasons, whereas gain and loss have to be interpreted separately. While loss of HR or HER2 is discussed to be caused by clonal selection or tumor dedifferentiation, gain cannot be explained that way. Due to the possibility of false-negative or false-positive results for HR expression, discordance should be interpreted with caution $(14,20,21)$. The evaluation in different laboratories, variables including time and type of tissue sampling and fixation, staining methods, antigen retrieval and misinterpretation by the observer may lead to less reproducibility $(5,21,22)$. In adjuvant trastuzumab trials, reproducibility of HER2 was only given in $85 \%$ when carried out in different laboratories (13).

Nevertheless, all HR and HER2 variations can not only be explained by preanalytical and analytical reasons. Supposing only technique and misinterpretation reasons, discordant results for at least ER and PR as well as gain and loss, which all were tested by IHC, should be the same.

Furthermore, there have already been some prospective study trials under almost perfect pathological conditions, which reported of only slightly less discordant results than the retrospective trials (ER 12.6\%, PR 31.2\% and HER2 $5.5 \%)(16,14)$. Apart from that, improvement of techniques during the last years should have minimized false results, although none of that has been observed (5).

Therefore, the theory of tumor's independent cell clones having different genetic profile is supported more and more $(6,12,23)$. Parallel clonal evolution of therapy-resistant distant seeding tumor cells or aberrant cells of unknown origin, as well as tumor heterogeneity have been discussed in previous studies $(12,15,24,25)$.

In the present study, several therapies, metastasis sites and patient characteristics have been analyzed concerning their association towards discordance.

Chemotherapy was significantly correlated with less HR concordance (subgroups $1 / 2$ ), whereas especially anthracyclines 
Table VII. Univariate logistic regression (ER/PR concordanceldiscordance).

\begin{tabular}{|c|c|c|c|c|c|c|c|c|c|c|c|c|}
\hline \multirow[t]{2}{*}{$\begin{array}{l}\text { Tumor biology/ } \\
\text { Subtype }\end{array}$} & \multicolumn{3}{|c|}{$\begin{array}{l}\text { ER/PR concordance } \\
\text { (113 patients) }\end{array}$} & \multicolumn{2}{|c|}{$\begin{array}{l}\text { ER/PR loss } \\
\text { ( } 69 \text { patients) }\end{array}$} & $\begin{array}{l}\text { ER+/PR loss } \\
\text { (43 patients) }\end{array}$ & \multicolumn{2}{|c|}{$\begin{array}{c}\text { ER concordance } \\
\text { (181 patients) }\end{array}$} & \multicolumn{2}{|c|}{$\begin{array}{c}\text { ER loss } \\
\text { (25 patients) }\end{array}$} & \multicolumn{2}{|c|}{$\begin{array}{c}\text { ER gain } \\
\text { (16 patients) }\end{array}$} \\
\hline & $\begin{array}{c}\text { OR } \\
(95 \% \mathrm{CI})\end{array}$ & $p$-Value & $\begin{array}{c}\text { OR } \\
(95 \% \mathrm{CI})\end{array}$ & $p$-Value & $\begin{array}{c}\mathrm{OR} \\
(95 \% \mathrm{CI})\end{array}$ & $p$-Value & $\begin{array}{c}\text { OR } \\
(95 \% \mathrm{CI})\end{array}$ & $p$-Value & $\begin{array}{c}\text { OR } \\
(95 \% \mathrm{CI})\end{array}$ & $p$-Value & $\underset{(95 \% \mathrm{CI})}{\mathrm{OR}} p$ & $p$-Value \\
\hline Age & $\begin{array}{c}0.989 \\
(0.967-1.012)\end{array}$ & 0.336 & $\begin{array}{c}1.007 \\
(0.983-1.032)\end{array}$ & 0.550 & $\begin{array}{c}1.001 \\
(0.973-1.030)\end{array}$ & 0.947 & $\begin{array}{c}0.999 \\
(0.971-1.028)\end{array}$ & 0.947 & $\begin{array}{c}1.015 \\
(0.980-1.051)\end{array}$ & 0.414 & $\begin{array}{c}0.975 \\
(0.933-1.020)\end{array}$ & 0.273 \\
\hline Interval & $\begin{array}{c}1.000 \\
(0.995-1.005)\end{array}$ & 0.996 & $\begin{array}{c}0.999 \\
(0.993-1.004)\end{array}$ & 0.648 & $\begin{array}{c}0.999 \\
(0.993-1.005)\end{array}$ & 0.754 & $\begin{array}{c}1.004 \\
(0.998-1.010)\end{array}$ & 0.197 & $\begin{array}{c}1.000 \\
(0.992-1.007)\end{array}$ & 0.910 & $\begin{array}{c}1.008 \\
(1.000-1.016)\end{array}$ & 0.042 \\
\hline Chemotherapy & $\begin{array}{c}0.467 \\
(0.260-0.838)\end{array}$ & 0.011 & $\begin{array}{c}2.205 \\
(1.125-4.323)\end{array}$ & 0.021 & $\begin{array}{c}1.622 \\
(0.749-3.515)\end{array}$ & 0.220 & $\begin{array}{c}0.321 \\
(0.128-0.804)\end{array}$ & 0.015 & $\begin{array}{c}2.585 \\
(0.852-7.842)\end{array}$ & 0.093 & $\begin{array}{c}3.374 \\
(0.745-15.274)\end{array}$ & 4) 0.114 \\
\hline Anthracyclines & $\begin{array}{c}0.460 \\
(0.269-0.788)\end{array}$ & 0.005 & $\begin{array}{c}2.002 \\
(1.112-3.604)\end{array}$ & 0.021 & $\begin{array}{c}2.048 \\
(1.015-4.133)\end{array}$ & 0.045 & $\begin{array}{c}0.469 \\
(0.229-0.964)\end{array}$ & 0.039 & $\begin{array}{c}1.341 \\
(0.575-3.131)\end{array}$ & 0.497 & $\begin{array}{c}4.088 \\
(1.131-14.774)\end{array}$ & 4) \\
\hline Taxanes & $\begin{array}{c}0.473 \\
(0.275-0.811)\end{array}$ & 0.007 & $\begin{array}{c}2.316 \\
(1.267-4.232)\end{array}$ & 0.006 & $\begin{array}{c}1.832 \\
(0.908-3.696)\end{array}$ & 0.091 & $\begin{array}{c}0.524 \\
(0.255-1.077)\end{array}$ & 0.079 & $\begin{array}{c}2.208 \\
(0.883-5.522)\end{array}$ & 0.090 & $\begin{array}{c}1.345 \\
(0.471-3.839)\end{array}$ & 0.580 \\
\hline Therapy lines & $\begin{array}{c}0.714 \\
(0.534-0.955)\end{array}$ & 0.023 & $\begin{array}{c}1.296 \\
(0.976-1.721)\end{array}$ & 0.073 & $\begin{array}{c}1.139 \\
(0.826-1.570)\end{array}$ & 0.427 & $\begin{array}{c}1.568 \\
(1.140-2.157)\end{array}$ & 0.006 & $\begin{array}{c}1.358 \\
(0.944-1.954)\end{array}$ & 0.099 & $\begin{array}{c}1.586 \\
(1.058-2.377)\end{array}$ & 0.026 \\
\hline $\begin{array}{l}\text { Endocrine } \\
\text { therapy }\end{array}$ & $\begin{array}{c}0.089 \\
(0.341-1.080)\end{array}$ & 0.089 & $\begin{array}{c}6.035 \\
(2.591-14.056)\end{array}$ & $<0.001$ & $\begin{array}{c}12.263 \\
(2.873-52.344)\end{array}$ & 0.001 & $\begin{array}{c}1.757 \\
(0.873-3.538)\end{array}$ & 0.114 & $\begin{array}{c}2.585 \\
(0.852-7.842)\end{array}$ & 0.093 & - & - \\
\hline $\begin{array}{l}\text { Aromatase } \\
\text { inhibitors }\end{array}$ & $\begin{array}{c}0.560 \\
(0.328-0.956)\end{array}$ & 0.034 & $\begin{array}{c}2.538 \\
(1.948-6.426)\end{array}$ & $<0.001$ & $\begin{array}{c}3.767 \\
(1.838-7.719)\end{array}$ & $<0.001$ & $\begin{array}{c}1.294 \\
(0.648-2.586)\end{array}$ & 0.465 & $\begin{array}{c}2.060 \\
(0.882-4.814)\end{array}$ & 0.095 & - & - \\
\hline Letrozol & $\begin{array}{c}0.750 \\
(0.377-1.317)\end{array}$ & 0.273 & $\begin{array}{c}3.291 \\
(1.725-6.280)\end{array}$ & $<0.001$ & $\begin{array}{c}3.071 \\
(1.507-6.256)\end{array}$ & 0.002 & $\begin{array}{c}1.108 \\
(0.490-2.503)\end{array}$ & 0.805 & $\begin{array}{c}2.015 \\
(0.832-4.876)\end{array}$ & 0.120 & - & - \\
\hline Tamoxifen & $\begin{array}{c}1.129 \\
(0.663-1.922)\end{array}$ & 0.656 & $\begin{array}{c}1.465 \\
(0.827-2.597)\end{array}$ & 0.191 & $\begin{array}{c}1.921 \\
(0.981-3.763)\end{array}$ & 0.057 & $\begin{array}{c}2.047 \\
(0.983-4.262)\end{array}$ & 0.056 & $\begin{array}{c}0.878 \\
(0.376-2.052)\end{array}$ & 0.765 & - & - \\
\hline GnRH analogs & $\begin{array}{c}0.754 \\
(0.286-1.989)\end{array}$ & 0.569 & $\begin{array}{c}1.457 \\
(0.540-3.936)\end{array}$ & 0.457 & $\begin{array}{c}1.680 \\
(0.565-4.997)\end{array}$ & 0.351 & $\begin{array}{c}1.891 \\
(0.417-8.567)\end{array}$ & 0.409 & $\begin{array}{c}0.984 \\
(0.212-4.555)\end{array}$ & 0.983 & - & - \\
\hline Fulvestrant & $\begin{array}{c}0.290 \\
(0.102-0.828)\end{array}$ & 0.021 & $\begin{array}{c}2.424 \\
(0.959-6.128)\end{array}$ & 0.061 & $\begin{array}{c}2.483 \\
(0.925-6.662)\end{array}$ & 0.071 & $\begin{array}{c}0.897 \\
(0.283-2.839)\end{array}$ & 0.853 & $\begin{array}{c}1.444 \\
(0.392-5.323)\end{array}$ & 0.581 & - & - \\
\hline $\begin{array}{l}\text { Radiation } \\
\text { therapy }\end{array}$ & $\begin{array}{c}1.572 \\
(0.271-9.122)\end{array}$ & 0.614 & $\begin{array}{c}3.000 \\
(0.525-17.159)\end{array}$ & 0.217 & $\begin{array}{c}3.182 \\
(0.344-29.432)\end{array}$ & 0.308 & $\begin{array}{c}0.512 \\
(0.208-1.262)\end{array}$ & 0.146 & $\begin{array}{c}1.615 \\
(0.166-15.723)\end{array}$ & 0.680 & $\begin{array}{c}0.724 \\
(0.065-8.054)\end{array}$ & 0.793 \\
\hline $\begin{array}{l}\text { Trastuzumab/ } \\
\text { Pertuzumab }\end{array}$ & $\begin{array}{c}0.563 \\
(0.259-1.223)\end{array}$ & 0.147 & $\begin{array}{c}2.378 \\
(1.100-5.145)\end{array}$ & 0.028 & $\begin{array}{c}1.550 \\
(0.640-3.753)\end{array}$ & 0.331 & $\begin{array}{c}0.492 \\
(0.207-1.167)\end{array}$ & 0.107 & $\begin{array}{c}2.803 \\
(1.061-7.408)\end{array}$ & 0.038 & $\begin{array}{c}0.872 \\
(0.188-4.038)\end{array}$ & 0.861 \\
\hline Metastas & & & & & & & & & & & & \\
\hline Lung & $\begin{array}{c}1.110 \\
(0.388-3.174)\end{array}$ & 0.845 & $\begin{array}{c}1.117 \\
(0.367-3.401)\end{array}$ & 0.845 & $\begin{array}{c}1.044 \\
(0.281-3.874)\end{array}$ & 0.949 & & & $\begin{array}{c}1.231 \\
(0.261-5.802)\end{array}$ & 0.793 & $\begin{array}{c}2.121 \\
(0.435-10.343)\end{array}$ & 3) \\
\hline Liver & $\begin{array}{c}0.908 \\
(0.490-1.683)\end{array}$ & 0.758 & $\begin{array}{c}1.190 \\
(0.617-2.294)\end{array}$ & 0.604 & $\begin{array}{c}1.121 \\
(0.521-2.415)\end{array}$ & 0.770 & & & $\begin{array}{c}1.277 \\
(0.502-3.246)\end{array}$ & 0.608 & $\begin{array}{c}0.434 \\
(0.095-1.975)\end{array}$ & 0.280 \\
\hline Bone & $\begin{array}{c}1.052 \\
(0.569-1.942)\end{array}$ & 0.872 & $\begin{array}{c}1.147 \\
(0.596-2.207)\end{array}$ & 0.681 & $\begin{array}{c}1.459 \\
(0.697-3.052)\end{array}$ & 0.316 & $\begin{array}{c}1.406 \\
(0.606-3.262)\end{array}$ & 0.428 & $\begin{array}{c}0.755 \\
(0.269-2.119)\end{array}$ & 0.594 & $\begin{array}{c}0701 \\
(0.192-2.560)\end{array}$ & 0.591 \\
\hline Thoracic wall & $\begin{array}{c}2.404 \\
(0.948-6.096)\end{array}$ & 0.065 & $\begin{array}{c}0.434 \\
(0.142-1.328)\end{array}$ & 0.143 & $\begin{array}{c}0.170 \\
(0.022-1.297)\end{array}$ & 0.087 & $\begin{array}{c}0.605 \\
(0.223-1.643)\end{array}$ & 0.324 & $\begin{array}{c}1.207 \\
(0.332-4.392)\end{array}$ & 0.775 & $\begin{array}{c}2.146 \\
(0.563-8.175)\end{array}$ & 0.263 \\
\hline LN other & $\begin{array}{c}0.857 \\
(0.344-2.197)\end{array}$ & 0.748 & $\begin{array}{c}1.327 \\
(0.498-3.531)\end{array}$ & 0.571 & $\begin{array}{c}1.121 \\
(0.353-3.566)\end{array}$ & 0.846 & $\begin{array}{c}0.836 \\
(0.262-2.664)\end{array}$ & 0.762 & $\begin{array}{c}1.543 \\
(0.416-5.718)\end{array}$ & 0.517 & $\begin{array}{c}0.696 \\
(0.087-5.580)\end{array}$ & 0.733 \\
\hline Other sites & $\begin{array}{c}0.719 \\
(0.394-1.312)\end{array}$ & 0.283 & $\begin{array}{c}0.893 \\
(0.464-1.718)\end{array}$ & 0.735 & $\begin{array}{c}0.965 \\
(0.451-2.068)\end{array}$ & 0.928 & $\begin{array}{c}1.119 \\
(0.510-2.454)\end{array}$ & 0.779 & $\begin{array}{c}0.679 \\
(0.243-1.901)\end{array}$ & 0.461 & $\begin{array}{c}1.312 \\
(0.436-3.951)\end{array}$ & 0.629 \\
\hline
\end{tabular}

showed association with less concordance of both ER and PR. Other publications have also showed a significant correlation between previous anthracycline therapy and ER discordance and a higher possibility of PR loss at recurrence under treatment with anthracyclines or taxanes $(8,7)$. In the present study, cases with ER and/or PR loss, as well as PR loss with concordant positive ER, were not associated with anthracycline or taxane treatment, but with ET, as has been reported consistently in previous studies (6). Interestingly, ET was correlated with a higher probability of persisting positive HR (group 1/2), whereas no significant result was calculated for less HR loss (group $1 / 2$ to $3 / 4$ ).

However, as mentioned above, ET was only associated with single receptor PR loss and not with ER loss. Patients with changing PR status (positive to negative) remain in group $1 / 2$. Since the 1980 s, this phenomenon is a point of interest for many studies. The theory of eliminated hormone sensitive cells, induced by ET, which leaves the more 
Table VIII. Results of multivariate logistic regression analysis (ER/PR concordance/discordance).

\begin{tabular}{|c|c|c|c|c|c|c|c|c|c|c|c|c|}
\hline \multirow[t]{2}{*}{$\begin{array}{l}\text { Tumor biology/ } \\
\text { Subtype }\end{array}$} & \multicolumn{3}{|c|}{$\begin{array}{l}\text { ER/PR concordance } \\
\text { (113 patients) }\end{array}$} & \multicolumn{2}{|c|}{$\begin{array}{l}\text { ER/PR loss } \\
\text { (69 patients) }\end{array}$} & $\begin{array}{l}\text { ER+/PR loss } \\
\text { (43 patients) }\end{array}$ & \multicolumn{2}{|c|}{$\begin{array}{c}\text { ER concordance } \\
\text { (181 patients) }\end{array}$} & \multicolumn{2}{|c|}{$\begin{array}{c}\text { ER loss } \\
\text { (25 patients) }\end{array}$} & \multicolumn{2}{|c|}{$\begin{array}{l}\text { ER gain } \\
\text { (16 patients) }\end{array}$} \\
\hline & $\begin{array}{c}\text { OR } \\
(95 \% \mathrm{CI})\end{array}$ & $p$-Value & $\begin{array}{c}\mathrm{OR} \\
(95 \% \mathrm{CI})\end{array}$ & $p$-Value & $\begin{array}{c}\mathrm{OR} \\
(95 \% \mathrm{CI})\end{array}$ & $p$-Value & $\begin{array}{c}\text { OR } \\
(95 \% \mathrm{CI})\end{array}$ & $p$-Value & $\begin{array}{c}\mathrm{OR} \\
(95 \% \mathrm{CI})\end{array}$ & $p$-Value & $\underset{(95 \% \mathrm{CI})}{\mathrm{OR}} p$ & $p$-Value \\
\hline Chemotherapy & & & & & & & $\begin{array}{c}0.460 \\
(0.149-1.417)\end{array}$ & 0.176 & & & & \\
\hline Anthracycline & $\begin{array}{c}0.414 \\
(0.237-0.723)\end{array}$ & 0.002 & & & & & & & & & $\begin{array}{c}2.967 \\
(0.739-11.906)\end{array}$ & 6) \\
\hline Therapy lines & & & & & & & $\begin{array}{c}0.754 \\
(0.504-1.129)\end{array}$ & 0.170 & & & $\begin{array}{c}1.347 \\
(0.837-2.168)\end{array}$ & 0.220 \\
\hline Endocrine therap & & ( & $\begin{array}{c}4.697 \\
(1.943-11.352)\end{array}$ & 2) & $\begin{array}{c}9.878 \\
(2.246-43.452)\end{array}$ & 0.002 & & & & & & \\
\hline Letrozol & & & $\begin{array}{c}2.037 \\
(1.031-4.024)\end{array}$ & 0.041 & $\begin{array}{c}1.795 \\
(0.859-3.750)\end{array}$ & 0.119 & & & & & & \\
\hline Tamoxifen & & & & & & & $\begin{array}{c}2.168 \\
(1.032-4.629)\end{array}$ & 0.041 & & & & \\
\hline Fulvestrant & $\begin{array}{c}0.255 \\
(0.085-0.765)\end{array}$ & 0.015 & & & & & & & & & & \\
\hline $\begin{array}{l}\text { Trastuzumab/ } \\
\text { Pertuzumab }\end{array}$ & & & & & & & $\begin{array}{c}2.803 \\
100.061-7.408\end{array}$ & 8) 0.038 & & & & \\
\hline $\begin{array}{l}\text { Metastasis site; } \\
\text { thoracic wall }\end{array}$ & $\begin{array}{c}2.831 \\
(1.088-7.363)\end{array}$ & 0.033 & & & & & & & & & & \\
\hline
\end{tabular}

aggressive hormone insensitive tumor cells, has been discussed. Sequential BC biopsies have shown that ER levels are slightly reduced under received ET, but no complete loss of expression was seen. This was more frequently observed for PR expression. Especially the impact of tamoxifen was illustrated, which possibly explains the correlation between ET and PR loss in the present study (62\% of patients who were treated with ET received tamoxifen) (26).

The PR gene seems to be highly sensitive to ET. Several mechanisms have been mentioned which could specifically lead to a genetic loss of PR $(19,27,28)$. Whether ET correlates with genetic alterations is not clear and needs to be further investigated.

Another point of interest was the treatment with T/P. These monoclonal antibodies seem to be associated with a higher probability of HR loss as well as single ER loss, HER2 loss and any tumor phenotype's subgroup discordance. Although the influence of T/P has been observed before, it should be interpreted with caution (8).

Patients with positive HER2 status in primary $\mathrm{BC}$ received both $\mathrm{T} / \mathrm{P}$ and chemotherapy in $65 \%$ of cases in the course of the time. As has been reported in previous studies, chemotherapy is especially associated with discordance of HER2 (29). Although the correlation of chemotherapy and discordance of HER2 was not observed in the present study, the combination of chemotherapy and T/P could bias single T/P's influence on HER2 change.
The group of patients who received T/P was associated with a higher probability of HR or single ER loss. About $26 \%$ of the patients with ER loss and $30 \%$ of the patients with $\mathrm{HR}$ received $\mathrm{T} / \mathrm{P}$.

Whether HER2 as well as HR discordance reflects the mechanism of therapy resistance or heterogeneity of expression within the primary site, is unclear (29). Rooijen et al. perceived an interesting fact: Patients who showed HER2 status discordance, had a higher HR expression as well (30). Further research is needed to prove whether this phenomenon of HER2 and HR correlation is due to analytical variations or a real genetic correlation.

Only a few authors have analyzed the correlation between changes of tumor biology and metastasis sites. Curtit et al. have reported a higher possibility of PR change in liver specimens (8). In the present study, significant results were calculated for the association between lymph node metastases and HER2 loss (except axillary lymph nodes; $p=0.026$ ) and between thoracic wall metastases and ER/PR concordance $(p=0.033)$. More prospective trials with similar results could have important therapeutic consequences. If specific metastasis sites showed specific concordance or discordance characteristics, this finding would help physicians to decide whether or not to carry out another biopsy.

Some studies have shown worse OS of positive HR change to negative HR, though direct comparison is not possible because of different subgroup classification $(6,11,15)$. 


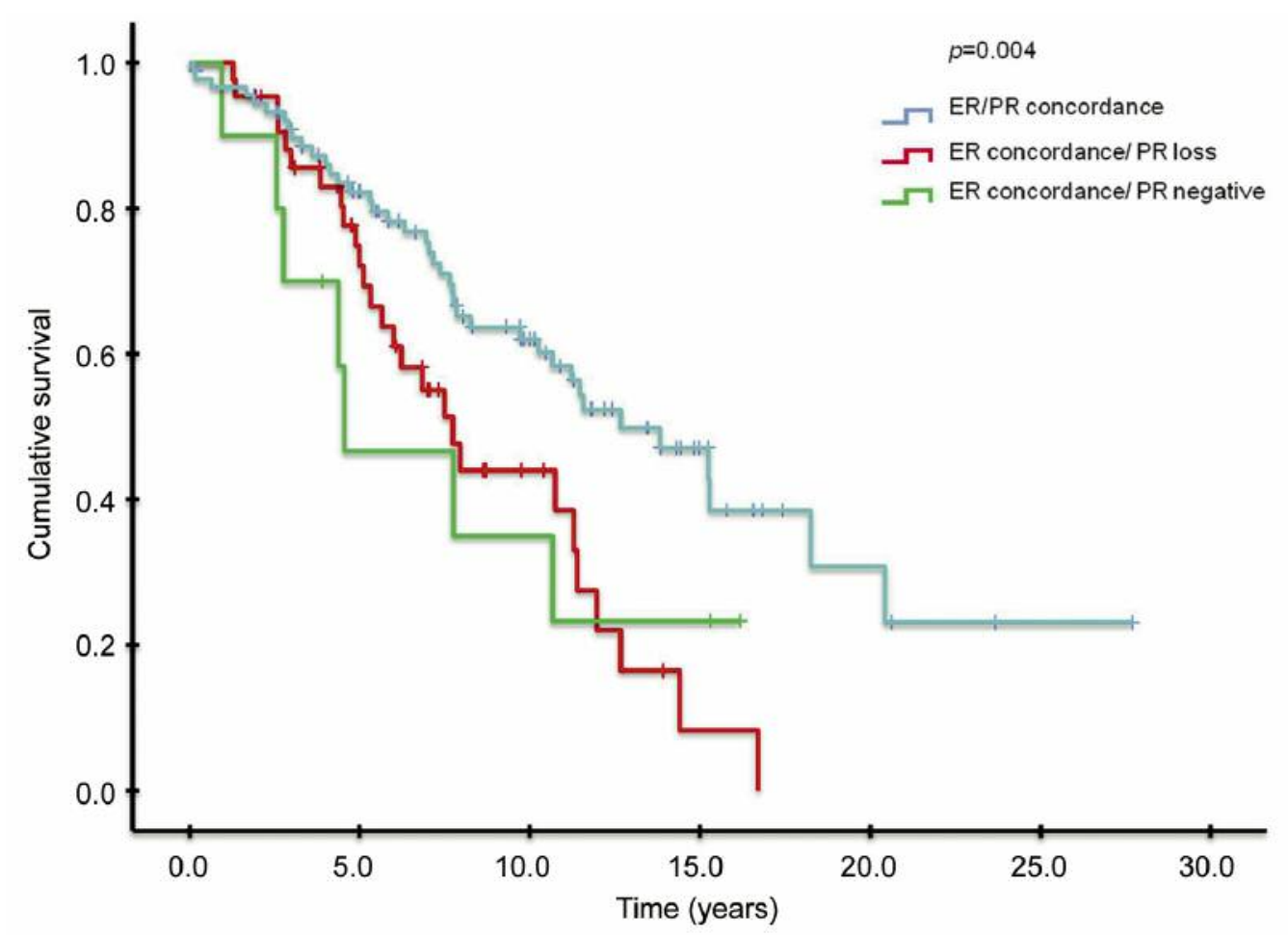

Figure 1. Overall survival $(E R / P R)$.

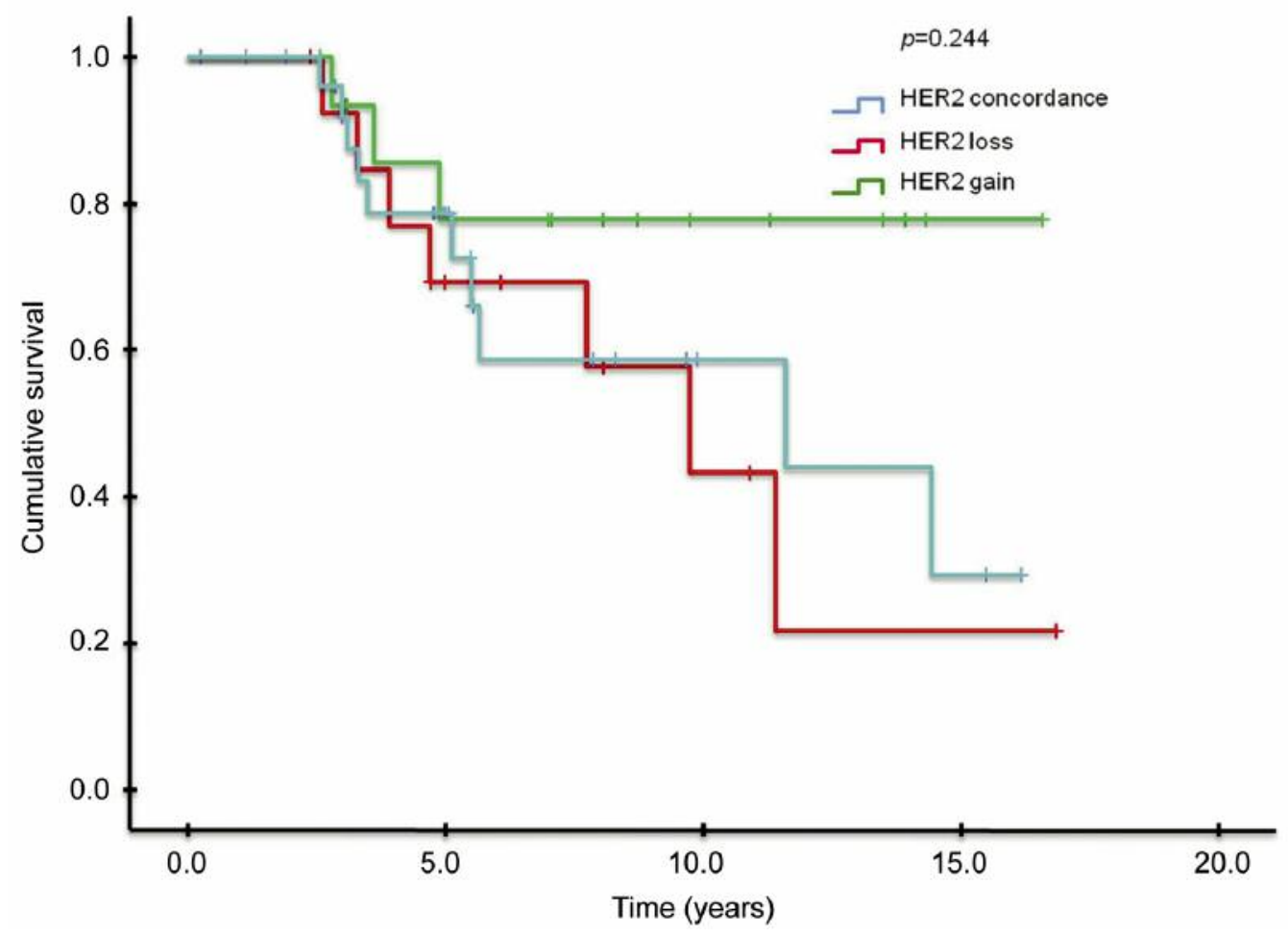

Figure 2. Overall survival (HER2). 


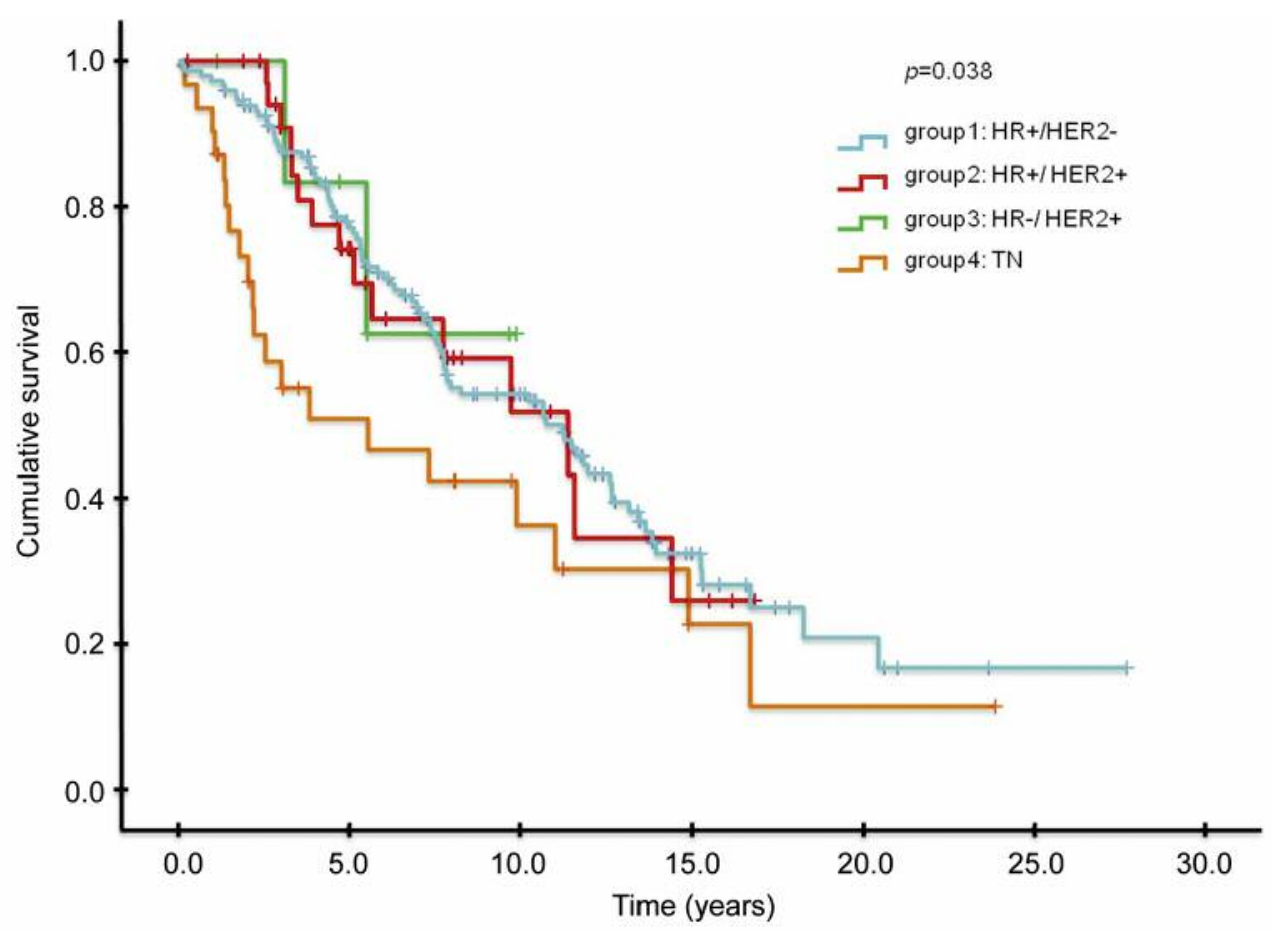

Figure 3. Overall survival (tumor phenotype).

In a study including 139 cases, patients whose tumor phenotype changed into triple negative subtype until breast cancer recurrence showed a worse prognosis in postrecurrence-survival and OS (11). Shiino et al. have presented a significant worse survival for the group which lost HR and/or HER2 status (15). Their subgroup classification is similar to that of the present study (group $1 / 2$ to $3 / 4$ ), whereas our study failed to prove any impact on survival for the change of tumor's phenotype (Table IX). Only patients with triple negative breast cancer (subgroup 4) showed worse survival rates, regardless of any changes in tumor biology (OS: $p=0.017$; PMS: $p=0.037$; Figure 3; Tables IX, XII).

Prospectively, Amir et al have not noticed any impact of discordance between primary and metastasized BC on survival. They have mentioned the possibility of suboptimal treatment in other study trials as a reason for poor survival in groups with discordant HR/HER2 (14).

By analyzing the survival of different variations of ER and PR expression, significant results were calculated. PR loss with concordant positive ER status showed a worse outcome in PMS ( $p=0.013$; Figure 1; Tables IX, X), just as PR at primary $\mathrm{BC}$ is significantly associated with worse OS as well as PMS ( $p=0.004$ and 0.044$)$, which is consistent with other studies $(6,28)$. Others, in turn, have not noticed such affection $(8,11)$.
The impact of HER2 status should be interpreted after mentioning some background. Various publications have illustrated worse outcomes for patients with HER2 loss $(11,25$, 29). In the present study, this phenomenon did not show any significant impact. Otherwise, patients with HER2 gain had a significantly improved prognosis for both PMS and OS in multivariate analysis $(p=0.002$ and 0.034 ), which could be due to directed treatment with T/P (Figure 2; Tables IX, XI). In contrast to previously published literature, HER2 discordance caused improved PMS $(p=0.005)$ which is probably due to the high number of patients with HER2 gain in comparison to HER2 loss (16 patients with HER2 gain; 14 patients with HER2 loss).

\section{Conclusion}

To sum up, the present study showed some significant results concerning chemotherapy, ET and T/P as influencing factors for receptor discordance. In our analysis, only PR at primary carcinoma, loss of PR and triple negative BC were relevant prognostic factors. All of these results highlight some problems that need to be solved in the future. Two facts emphasize the importance of continuing to improve and standardize preanalytical and analytical methods, as well as patients' treatment.

First, the limited treatment options in the triple negative subgroup could explain its worse prognosis. Second, the 
Table IX. OS and PMS.

\begin{tabular}{lcccc}
\hline & & & & PMS \\
\cline { 2 - 5 } Prognostic factors & HR $(95 \% \mathrm{CI})$ & $p$-Value & HR (95\%CI) & $p$-Value \\
\hline Group 1 & $0.770(0.521-1.136)$ & 0.188 & $1.026(0.695-1.513)$ & 0.898 \\
Group 2 & $0.921(0.535-1.585)$ & 0.766 & $0.710(0.413-1.221)$ & 0.216 \\
Group 3 & $0.782(0.192-3.177)$ & 0.731 & $0.416(0.103-1.684)$ & 0.219 \\
Group 4 & $1.794(1.108-2.904)$ & 0.017 & $1.669(1.030-2.702)$ & 0.037 \\
Discordance & $0.751(0.499-1.131)$ & 0.170 & $0.642(0.426-0.967)$ & 0.034 \\
ER/PR concordance & $0.768(0.536-1.101)$ & 0.151 & $0.706(0.491-1.013)$ & 0.059 \\
ER/PR positive-multivariate & $0.546(0.373-0.799)$ & 0.002 & $0.535(0.364-0.786)$ & 0.001 \\
ER and/or PR loss-multivariate & $0.925(0.494-1.732)$ & 0.807 & & \\
ER concordance/PR loss-multivariate & $1.402(0.958-2.053)$ & 0.082 & $1.474(1.010-2.151)$ & 0.044 \\
ER (primary BC) & $1.478(0.958-2.282)$ & 0.077 & $1.732(1.124-2.667)$ & 0.013 \\
PR (primary BC)-multivariate & $1.942(0.994-3.796)$ & 0.052 & $2.479(1.267-4.850)$ & 0.008 \\
HER2 (primary BC)-multivariate & $1.391(0.893-2.165)$ & 0.144 & $1.268(0.815-1.972)$ & 0.292 \\
HER2 discordance & $1.741(1.190-2.548)$ & 0.004 & $0.049(1.010-2.166)$ & 0.044 \\
HER2 loss & $1.947(1.039-3.649)$ & 0.038 & $1.579(0.944-2.641)$ & 0.082 \\
HER2 gain-multivariate & $1.117(0.666-1.872)$ & 0.675 & $1.980(1.177-3.329)$ & 0.010 \\
& $0.573(0.315-1.040)$ & 0.067 & $0.424(0.233-0.770)$ & 0.005 \\
& $1.038(0.483-2.231)$ & 0.924 & $0.755(0.351-1.621)$ & 0.471 \\
\end{tabular}

Table X. OS of ER/PR.

\begin{tabular}{lcc}
\hline ER/PR & Median OS (years) & SD with 95\%CI \\
\hline ER/PR concordant & 12.7 & $1.900(8.956-16.419)$ \\
ER concordant/PR loss & 7.7 & $0.876(6.026-9.459)$ \\
ER positive/PR negative & 4.6 & $2.404(0.000-9.271)$ \\
Cox regression & $p=0.004$ & OR=0.723 $(95 \%$ CI $=0.419-1.248)$ \\
\hline
\end{tabular}

Table XI. OS of HER2.

\begin{tabular}{lcc}
\hline HER2 & Median OS (years) & SD with 95\%CI \\
\hline HER2 concordance & 11.6 & $6.268(0.000-23.874)$ \\
HER2 loss & 9.7 & $2.321(5.185-14.283)$ \\
HER2 gain & Mean survival: 13.7 & $1.450(10.896-16.582)$ \\
Cox regression & $p=0.244$ & OR $=1.632(95 \% \mathrm{CI}=1.175-2.268)$ \\
\hline
\end{tabular}

Table XII. OS of tumor phenotype.

\begin{tabular}{lcc}
\hline Tumor phenotype & Median OS (years) & SD with 95\%CI \\
\hline Group 1 & 10.7 & $1.662(7.491-14.005)$ \\
Group 2 & 11.4 & $2.780(5.948-16.847)$ \\
Group 3 & Mean survival: 7.8 & $1.172(5.548-10.141)$ \\
Group 4 & 5.5 & $3.273(0.000-11.961)$ \\
Cox regression & $p=0.038$ & OR $=1.190(95 \% \mathrm{CI}=1.010-1.401)$ \\
\hline
\end{tabular}


slightly higher rates of this study's receptor discordance in comparison to prospective studies, as well as the unexpected increased number of cases with HER2 gain than HER2 loss could be partly explained by laboratory differences. With no established genetic cause explaining the loss of PR while ER remains positive, we encourage further research on the phenomenon. Other facts that need to be investigated in the future are the correlation of $\mathrm{HR}$ and $\mathrm{T} / \mathrm{P}$ as well as the possibility that thoracic wall and lymph node metastases (except axillary $\mathrm{LN}$ ) show characteristics concerning tumor phenotype's concordance or discordance.

\section{Conflicts of Interest}

The Authors declare that they have no conflicts of interest.

\section{Authors' Contributions}

Manuscript writing: CV, VK and FT; Manuscript editing: BM, SL, JJV; Statistical analysis: CV, SH; Data collection: WM, SL, BM; Project development: CV, BM, VK and FT.

\section{References}

1 Fitzmaurice C, Allen C, Barber RM, Barregard L, Bhutta ZA, Brenner H, Dicker DJ, Chimed-Orchir O, Dandona R, Dandona L, Fleming T, Forouzanfar MH, Hancock J, Hay RJ, HunterMerrill R, Huynh C, Hosgood HD, Johnson CO, Jonas JB, Khubchandani J, Kumar GA, Kutz M, Lan Q, Larson HJ, Liang X, Lim SS, Lopez AD, MacIntyre MF, Marczak L, Marquez N et al: Global, regional, and national cancer incidence, mortality, years of life lost, years lived with disability, and disabilityadjusted life-years for 32 cancer groups, 1990 to 2015: A systematic analysis for the global burden of disease study. JAMA Oncol 3: 524-548, 2017. PMID: 27918777. DOI: 10.1001/jamaoncol.2016.5688

2 Cardoso F, Spence D, Mertz S, Corneliussen-James D, Sabelko K, Gralow J, Cardoso M-J, Peccatori F, Paonessa D, Benares A, Sakurai N, Beishon M, Barker S-J and Mayer M: Global analysis of advanced/metastatic breast cancer: Decade report (2005-2015). Breast (Edinburgh, Scotland) 39: 131-138, 2018. PMID: 29679849. DOI: 10.1016/j.breast.2018.03.002

3 Malvezzi M, Carioli G, Bertuccio P, Boffetta P, Levi F, La Vecchia $\mathrm{C}$ and Negri E: European cancer mortality predictions for the year 2018 with focus on colorectal cancer. Ann Oncol 29: 1016-1022, 2018. PMID: 29562308. DOI: 10.1093/annonc/ mdy033

4 Untch M, Würstlein R, Marschner N, Lüftner D, Augustin D, Briest S, Ettl J, Haidinger R, Müller L, Müller V, Ruckhäberle $\mathrm{E}$, Harbeck $\mathrm{N}$ and Thomssen $\mathrm{C}$ : 4th International Consensus Conference on Advanced Breast Cancer (ABC4), Lisbon, November 4, 2017 *: ABC4 Consensus: Assessment by a panel of German Experts. Geburtshilfe und Frauenheilkunde 78: 469480, 2018. PMID: 29880982. DOI: 10.1055/a-0594-2243

5 Aurilio G, Disalvatore D, Pruneri G, Bagnardi V, Viale G, Curigliano G, Adamoli L, Munzone E, Sciandivasci A, Vita F de, Goldhirsch A and Nolè F: A meta-analysis of oestrogen receptor, progesterone receptor and human epidermal growth factor receptor 2 discordance between primary breast cancer and metastases. Eur J Cancer 50: 277-289, 2014. PMID: 24269135. DOI: 10.1016/j.ejca.2013.10.004

6 Bogina G, Bortesi L, Marconi M, Venturini M, Lunardi G, Coati F, Massocco A, Manfrin E, Pegoraro C and Zamboni G: Comparison of hormonal receptor and HER-2 status between breast primary tumours and relapsing tumours: clinical implications of progesterone receptor loss. Virchows Archiv 459: 1-10, 2011. PMID: 21643691. DOI: 10.1007/s00428-011-1097-7

7 Ongaro E, Gerratana L, Cinausero M, Pelizzari G, Poletto E, Giangreco M, Andreetta C, Pizzolitto S, Di Loreto C, Minisini AM, Mansutti M, Russo S, Fasola G and Puglisi F: Comparison of primary breast cancer and paired metastases: biomarkers discordance influence on outcome and therapy. Future Oncol 14: 849-859, 2018. PMID: 29527957. DOI: 10.2217/fon-2017-0384, 03.05.2018

8 Curtit E, Nerich V, Mansi L, Chaigneau L, Cals L, Villanueva C, Bazan F, Montcuquet P, Meneveau N, Perrin S, Algros M-P and Pivot $\mathrm{X}$ : Discordances in estrogen receptor status, progesterone receptor status, and HER2 status between primary breast cancer and metastasis. Oncologist 18: 667-674, 2013. PMID: 23723333. DOI: 10.1634/theoncologist.2012-0350

9 Shachar SS, Mashiach T, Fried G, Drumea K, Shafran N, Muss HB and Bar-Sela G: Biopsy of breast cancer metastases: patient characteristics and survival. BMC Cancer 17: 7, 2017. PMID: 28052766. DOI: $10.1186 / \mathrm{s} 12885-016-3014-6$

10 Tobin NP, Harrell JC, Lövrot J, Egyhazi Brage S, Frostvik Stolt M, Carlsson L, Einbeigi Z, Linderholm B, Loman N, Malmberg M, Walz T, Fernö M, Perou CM, Bergh J, Hatschek T and Lindström LS: Molecular subtype and tumor characteristics of breast cancer metastases as assessed by gene expression significantly influence patient post-relapse survival. Ann Oncol 26: 81-88, 2015. PMID: 25361981. DOI: 10.1093/annonc/ mdu498

11 Dieci MV, Barbieri E, Piacentini F, Ficarra G, Bettelli S, Dominici M, Conte PF and Guarneri V: Discordance in receptor status between primary and recurrent breast cancer has a prognostic impact: a single-institution analysis. Ann Oncol 24: 101-108, 2013. PMID: 23002281. DOI: 10.1093/annonc/mds248

12 Kulka J, Székely B, Lukács LV, Kiss O, Tőkés AM, Vincze E, Turányi E, Fillinger J, Hanzély Z, Arató G, Szendrói M, Győrffy $B$ and Szász AM: Comparison of predictive immunohistochemical marker expression of primary breast cancer and paired distant metastasis using surgical material: A practice-based study. J Histochem Cytochem 64: 256-267, 2016. PMID: 27029768. DOI: 10.1369/0022155416639013

13 Liedtke C, Broglio K, Moulder S, Hsu L, Kau S-W, Symmans WF, Albarracin C, Meric-Bernstam F, Woodward W, Theriault RL, Kiesel L, Hortobagyi GN, Pusztai L and Gonzalez-Angulo AM: Prognostic impact of discordance between triple-receptor measurements in primary and recurrent breast cancer. Ann Oncol 20: 1953-1958, 2009. PMID: 19596702. DOI: 10.1093/annonc/ $\operatorname{mdp} 263$

14 Amir E, Miller N, Geddie W, Freedman O, Kassam F, Simmons C, Oldfield M, Dranitsaris G, Tomlinson G, Laupacis A, Tannock IF and Clemons M: Prospective study evaluating the impact of tissue confirmation of metastatic disease in patients with breast cancer. J Clin Oncol 30: 587-592, 2012. PMID: 22124102. DOI: $10.1200 / J C O .2010 .33 .5232$ 
15 Shiino S, Kinoshita T, Yoshida M, Jimbo K, Asaga S, Takayama $\mathrm{S}$ and Tsuda H: Prognostic impact of discordance in hormone receptor status between primary and recurrent sites in patients with recurrent breast cancer. Clin Breast Cancer 16: e133-40, 2016. PMID: 27268749. DOI: 10.1016/j.clbc.2016.05.014.

16 Amir E, Clemons M, Purdie CA, Miller N, Quinlan P, Geddie W, Coleman RE, Freedman OC, Jordan LB and Thompson AM: Tissue confirmation of disease recurrence in breast cancer patients: pooled analysis of multi-centre, multi-disciplinary prospective studies. Cancer Treat Rev 38: 708-714, 2012. PMID: 22178456. DOI: 10.1016/j.ctrv.2011.11.006

17 Lower EE, Khan S, Kennedy D and Baughman RP: Discordance of the estrogen receptor and HER-2/neu in breast cancer from primary lesion to first and second metastatic site. Breast Cancer Res Treat 90: 65-70, 2005. PMID: 15770528. DOI: 10.1007/ s10549-004-2756-z

18 Nishimura R, Osako T, Okumura Y, Tashima R, Toyozumi Y and Arima N: Changes in the ER, PgR, HER2, p53 and Ki-67 biological markers between primary and recurrent breast cancer: discordance rates and prognosis. World J Surg Oncol 9: 131, 2011. PMID: 22004841. DOI: 10.1186/1477-7819-9-131

19 Broom RJ, Tang PA, Simmons C, Bordeleau L, Mulligan AM, O'Malley FP, Miller N, Andrulis IL, Brenner DM and Clemons MJ: Changes in estrogen receptor, progesterone receptor and Her-2/neu status with time: discordance rates between primary and metastatic breast cancer. Anticancer Res 29: 1557-1562, 2009. PMID: 19443366.

20 Perez EA, Suman VJ, Davidson NE, Martino S, Kaufman PA, Lingle WL, Flynn PJ, Ingle JN, Visscher D and Jenkins RB: HER2 testing by local, central, and reference laboratories in specimens from the North Central Cancer Treatment Group N9831 intergroup adjuvant trial. J Clin Oncol 24: 3032-3038, 2006. PMID: 16809727. DOI: 10.1200/JCO.2005.03.4744

21 Pusztai L, Viale G, Kelly CM and Hudis CA: Estrogen and HER-2 receptor discordance between primary breast cancer and metastasis. Oncologist 15: 1164-1168, 2010. PMID: 21041379. DOI: $10.1634 /$ theoncologist.2010-0059

22 Nassar A: Core needle biopsy versus fine needle aspiration biopsy in breast - a historical perspective and opportunities in the modern era. Diagn Cytopathol 39: 380-388, 2011. PMID: 20949457. DOI: $10.1002 / \mathrm{dc} .21433$

23 Rossi S, Basso M, Strippoli A, Dadduzio V, Cerchiaro E, Barile R, D'Argento E, Cassano A, Schinzari G and Barone C: Hormone receptor status and HER2 expression in primary breast cancer compared with synchronous axillary metastases or recurrent metastatic disease. Clin Breast Cancer 15: 307-312, 2015. PMID: 25922284. DOI: 10.1016/j.clbc.2015.03.010
24 Videira M, Reis RL and Brito MA: Deconstructing breast cancer cell biology and the mechanisms of multidrug resistance. Biochim Biophys Acta 1846: 312-325, 2014. PMID: 25080053. DOI: $10.1016 /$ j.bbcan.2014.07.011

25 Demeulemeester J, Kumar P, Møller EK, Nord S, Wedge DC, Peterson A, Mathiesen RR, Fjelldal R, Zamani Esteki M, Theunis K, Fernandez Gallardo E, Grundstad AJ, Borgen E, Baumbusch LO, Børresen-Dale AL, White KP, Kristensen VN, van Loo $P$, Voet $T$ and Naume $B$ : Tracing the origin of disseminated tumor cells in breast cancer using single-cell sequencing. Genome Biol 17: 250, 2016. PMID: 27931250. DOI: $10.1186 / \mathrm{s} 13059-016-1109-7$

26 Gross GE, Clark GM, Chamness GC and McGuire WL: Multiple progesterone receptor assays in human breast cancer. Cancer Res 44: 836-840, 1984. PMID: 6692382.

27 Lapidus RG, Nass SJ and Davidson NE: The loss of estrogen and progesterone receptor gene expression in human breast cancer. J Mammary Gland Biol Neoplasia 3: 85-94, 1998. PMID: 10819507. DOI: 10.1023/A:1018778403001

28 Cui X, Schiff R, Arpino G, Osborne CK and Lee AV: Biology of progesterone receptor loss in breast cancer and its implications for endocrine therapy. J Clin Oncol 23: 7721-7735, 2005. PMID: 16234531. DOI: 10.1200/JCO.2005.09.004

29 Niikura N, Liu J, Hayashi N, Mittendorf EA, Gong Y, Palla SL, Tokuda Y, Gonzalez-Angulo AM, Hortobagyi GN and Ueno NT: Loss of human epidermal growth factor receptor 2 (HER2) expression in metastatic sites of HER2-overexpressing primary breast tumors. J Clin Oncol 30: 593-599, 2012. PMID: 22124109. DOI: 10.1200/JCO.2010.33.8889

30 van Rooijen JM, Munck L de, Graaf JC de, Siesling S, Vries EG de and Boers JE: Limited human epidermal growth factor receptor 2 discordance in metastatic breast cancer patients treated with trastuzumab, a population based study. Eur J Cancer 50: 885-891, 2014. PMID: 24491395. DOI: 10.1016/j.ejca.2014. 01.010
Received March 30, 2019

Revised April 23, 2019

Accepted April 24, 2019 